\title{
Estereotipos negativos de la vejez en personal de salud de un Hospital de la Ciudad de Querétaro, México
}

\author{
MIREYA FRANCO S., ENRIQUE VILLARREAL R. ${ }^{1}$, EMMA R. VARGAS D. ${ }^{\text {a }}$, \\ LIDIA MARTÍNEZ G. ${ }^{\text {la }}$, LILIANA GALICIA R. ${ }^{1 b}$
}

'Unidad de Investigación Epidemiológica y en Servicios de Salud Querétaro. Instituto Mexicano del Seguro Social. Querétaro, México. ${ }^{2}$ Unidad de Medicina Familiar No. 16 "Dr. Arturo Guerrero Ortiz". Querétaro. Instituto Mexicano del Seguro Social. Querétaro, México.

aLicenciada en Enfermería

bicenciada en Sistemas.

Recibido el 11 de marzo de 2009, aceptado el 20 de julio de 2010.

Correspondencia a:

Mireya Franco Saldaña. Dirección Sierra de las Vírgenes No. 116, Colonia. Villas del Sol. 76046. Querétaro. Qro. México.

Teléfono y Fax: 4422112300 ext. 51113

E-mail: yvani98@hotmail.com

\section{Prevalence of negative stereotypes towards old age among personnel of a general hospital}

Background: Social representations are value systems. Social stereotypes are a social consensus of traits associated with a specific group. Stereotypes about older subjects, generally have negative connotations. Aim: To assess the prevalence of negative stereotypes towards older subjects among health care personnel. Material and Methods: A questionnaire about stereotypes towards old age, with Likert- type questions that included health, social motivations and personality-character domains, was applied to 52 doctors, 12 residents, 80 general nurses, 36 auxiliary nurses, four social workers and 10 medical assistants, working at a general hospital. Results: The mean age of the professionals who answered the questionnaire was 38 years (95\% confidence intervals (CI) 37-39 years) and 78\% were women. The prevalence of a Global negative stereotype was 65.0\%(95\% CI: 59.5-70.5). The figure for the health domain was 64.5\% (IC95\%; 59.0-70.0), for the social motivation domain was 60\% (5\% CI: 54.3-65.7) and for the character-personality domain was 64\% (95\%CI: 58.4-69.6). Conclusions: There is a high prevalence of a negative stereotype towards old age among health care personnel.

(Rev Med Chile 2010; 138: 988-993).

Key words: Delivery of health care; Old age assistance; Stereotyped behavior.
$\mathrm{D}$ urante los últimos años se ha producido un aumento vertiginoso de las personas mayores de 65 años lo que ha hecho surgir un nuevo concepto llamado "envejecimiento generacional". México se encuentra ante esta situación, presenta un proceso acelerado de transición demográfica, transformándose en un país con más viejos que niños. Entre los años 2000 y 2050 la proporción de adultos mayores pasará de $7 \mathrm{al} 28 \%$ en una población de 130 millones en el año 2050, con el consecuente impacto en los servicios de salud ya que la población geriátrica se caracteriza por la alta demanda de servicios de salud ${ }^{1-3}$.

El proceso de envejecer denota una carga negativa, decadente e involutiva y desde una perspectiva biologicista se pone el énfasis en el deterioro y las pérdidas ${ }^{4}$.

Los gerontólogos han acuñado el término "edadismo" para referirse al concepto peyorativo de alguien basado en su edad avanzada ${ }^{5}$, lo que implica una visión estereotipada sobre las personas mayores por ser diferentes a las demás en sus opiniones, en la expresión, en la necesidad de afecto y en relación a su físico ${ }^{6,7}$.

Dentro de las representaciones sociales uno de los conceptos que mayor interés ha despertado en la Psicología Social es el de los estereotipos sociales así como sus relaciones con los prejuicios y la discriminación ${ }^{8}$.

Existen distintas definiciones de estereotipo: se 
ha identificado como un conjunto de atributos o características asignados a un grupo social ; como "imágenes que tenemos en la cabeza" o "cuadros de cabecera" que nos hablan del mundo antes de conocerlo"; como "una colección de rasgos sobre los que un gran porcentaje de gente concuerda como apropiados para describir a alguna clase de personas" 10 como "una proposición rígidamente establecida que atribuye una característica a todos los miembros de una categoría"; como el grado en que una característica está presente en un grupo de individuos ${ }^{11}$; como "una creencia exagerada que está asociada a una categoría ${ }^{12}$; o como consenso de opinión acerca de los rasgos atribuidos al grupo ${ }^{13}$.

Más allá de la definición se pueden señalar que en todas ellas se identifica un consenso social en relación a conjunto de rasgos asociados a un grupo o categoría social ${ }^{14,15}$. No obstante existen autores que suprimen la noción de consenso e incorporan la noción de pensamiento defectuoso ${ }^{16}$.

La importancia de identificar el estereotipo no se limita a la función cognitiva, se extiende a la función defensiva al emplearse para mantener y defender la posición en la sociedad. Esta función defensiva no es únicamente aplicable a los individuos, sino que sirve a su vez para que ciertos grupos mantengan su posición dominante sobre otros $^{17}$.

La sociedad se estratifica por edades, con expectativas y roles determinados por tradiciones y funciones sociales aprendidas, las cuales se incorporan al sistema de creencias para jugar un papel determinante al establecer escenarios para que la persona actúe de acuerdo a las expectativas de comportamiento generalizado. Estas creencias generalizadas acerca de rasgos que se suponen son típicos o característicos de determinados grupos sociales basados en informaciones ambiguas e incompletas son los estereotipos ${ }^{18}$.

En el sujeto anciano el estereotipo tiene por lo general una connotación negativa, tanto dentro de la sociedad en general, como dentro del mismo grupo de personas mayores ${ }^{18}$. Un estereotipo negativo sobre la vejez no es un tema reciente, hace más de cinco décadas ya se identificaba como un hallazgo consistente y estable ${ }^{19,20}$.

La investigación en el campo de los estereotipos sobre la vejez incluye la percepción de diferentes grupos; en familiares y cuidadores de adultos mayores existe una falsa concepción ${ }^{19,21}$; en jóvenes se percibe a la vejez como un período caracterizado por mala salud, inseguridad económica, soledad y decadencia en las capacidades físicas y menta$l^{2 s^{22,23}}$; en otros trabajos se ha identificado además de la edad, la clase social ${ }^{24}$.

Pero también existen estudios que hablan de la percepción positiva que sobre los ancianos tienen otros grupos ${ }^{25,26}$, específicamente en estudiantes la percepción positiva se orienta al dominio personal-expresivo ${ }^{27}$.

En los profesionales de la salud se ha señalado que tienen dificultad para reconocer la presencia de procesos patológicos en las personas mayores ya que asocian de forma natural la edad con la fragilidad y la debilidad (Montorio e Izal 1994) ${ }^{28}$ y se ha dicho que pueden llegar a utilizar la edad como un indicador para explicar el comportamiento, variando el diagnóstico y las recomendaciones para el tratamiento de las personas mayores en comparación con adultos de menor edad ${ }^{29}$. Y contrario a lo esperado se ha encontrado que el mayor contacto de la relación médico paciente no siempre elimina o reduce los estereotipos asociados a la edad ${ }^{30}$.

Las escalas que se han utilizado para medir los estereotipos incluyen las de actitudes, que evalúan el grado de disposición permanente respecto a determinadas cuestiones ${ }^{31}$. El cuestionario de evaluación de estereotipos negativos hacia la vejez $(\mathrm{CENVE})^{32}$ conjunta dos propuestas incorpora el factor salud mental al factor salud, y mantiene el factor carácter-personalidad y motivación-social siendo este un instrumento más integrador.

Si bien se ha evaluado el estereotipo en la población general y en médicos ${ }^{33-35}$ no existen estudios que analicen el estereotipo en el personal de salud por lo que el objetivo de este estudio es determinar la prevalencia de los estereotipos negativos de la vejez en el personal de salud.

\section{Material y Método}

Se diseñó un estudio transversal descriptivo en personal de salud, realizado en enero de 2008 en el Hospital General Regional No 1, del Instituto Mexicano del Seguro Social (IMSS), en el Estado de Querétaro, México.

La población estudiada incluyó médicos adscritos, médicos residentes, médicos internos, enfermeras generales, auxiliares de enfermería, trabajadoras sociales y asistentes médicas. Se excluyó personal directivo y administrativo. 
Estereotipos negativos de la vejez en personal de salud en México - M. Franco S. et al

Se distribuyó proporcionalmente el tamaño de la muestra entre el tipo de profesionales estudiado: médicos adscritos 52, médicos residentes 12 , médicos internos 6 , enfermeras generales 80 , auxiliares de enfermería 36 , trabajadoras sociales 4 y asistentes médicas 10 . Al interior de cada categoría se entrevistó al personal que se encontraba en el servicio el día que se realizó la encuesta, con técnica no aleatoria. Se eligió un día específico para realizar la encuesta a cada uno de los tipos de profesionistas incluidos, el día elegido se acudió al servicio y previo consentimiento informado se entrevistó al personal que en ese momento se encontraba y accedió a responder la encuesta. En todos los casos el Investigador principal mediante entrevista directa aplicó el cuestionario CENVE.

\section{Instrumentos}

El estereotipo negativo de la vejez se midió a través del instrumento CENVE que consta de un cuestionario de evaluación de estereotipos negativos hacia la vejez ${ }^{32}$, que se constituye de 15 preguntas, cada pregunta medido con escala likert del 1 al 4 (categorías: 1= muy en desacuerdo, 2 = algo en desacuerdo, 3 = algo de acuerdo, $4=$ muy de acuerdo). Se evaluaron tres dimensiones: dimensión salud, dimensión motivación-social y dimensión carácter-personalidad. Para cada una de las dimensiones la puntuación mínima de 5 y máxima 20, considerándose estereotipo negativo cuando la puntuación se identificó entre 12,5 y 20. La dimensión salud incluyó las preguntas 1 , 4, 7, 10 y 13; la dimensión motivación-social las preguntas 2, 5, 8, 11 y 14 ; y la dimensión carácterpersonalidad las preguntas $3,6,9,12$ y 15 . La evaluación global del estereotipo con mínimo de puntuación de 15 y máxima 60, se considera estereotipo negativo cuando la puntuación de encontró entre 37,5 y 60 (categorías 3 y 4).

\section{Estadística}

El tamaño de la muestra se calculó con la fórmula de porcentajes para población finita, utilizando la hipótesis de que la prevalencia del estereotipo negativo es superior al 70\% (prevalencia), $\mathrm{N}=1.295$ (población), $\mathrm{Z}=1,64$ (nivel de significancia), $\mathrm{d}=0,05$ (margen de error), $\mathrm{n}=$ 200 (tamaño de muestra). Se analizaron variables sociodemográficas (edad, género, categoría, antigüedad laboral y tipo de servicio). Se realizó en el programa SPSS e incluyó promedios, porcentajes e intervalos de confianza de $95 \%$.

\section{Resultados}

La edad promedio de la población estudiada es de 38, 07 años (IC 95\%, 37,06- 39,08) y la antigüedad laboral corresponde a 13,04 años (IC 95\%, 11,99-14,09). Predomina el sexo femenino con $78,0 \%$ (IC 95\%, 73,2-82,8).

La prevalencia del prejuicio de categorías algo de acuerdo y muy de acuerdo en la dimensión salud es de $46,5 \%$ y $18,0 \%$ respectivamente, para la dimensión motivación-social $45,0 \%$ y $15,0 \%$ y para la dimensión) carácter-personalidad de $42,0 \%$ y $22,0 \%$. Tablas 1,2 y 3 .

En la evaluación global, la prevalencia del prejuicio de categoría algo de acuerdo es de $45,5 \%$ y muy de acuerdo 19,5\%. Tabla 4 .

La prevalencia del estereotipo negativo global es de $65,0 \%$, para la dimensión salud $64,5 \%$, para motivación-social $60,0 \%$ y para carácterpersonalidad 64,0\%. Tabla 5.

Tabla 1. Prevalencia del prejuicio de categorías de la dimensión salud en personal de salud

\begin{tabular}{|lccc|}
\hline $\begin{array}{l}\text { Prejuicio de } \\
\text { categoría }\end{array}$ & $\%$ & \multicolumn{2}{c|}{$\begin{array}{c}\text { Intervalo de } \\
\text { confianza 95\% }\end{array}$} \\
\hline Muy en desacuerdo & 3,0 & 1,0 & 5,0 \\
Mínimo & Máximo \\
\hline Algo en desacuerdo & 32,5 & 27,1 & 37,9 \\
\hline Muy de de acuerdo & 46,5 & 40,7 & 52,3 \\
\hline
\end{tabular}


Estereotipos negativos de la vejez en personal de salud en México - M. Franco S. et al

Tabla 2. Prevalencia del prejuicio de categorías de la dimensión motivación-social en personal de salud

\begin{tabular}{|c|c|c|c|}
\hline \multirow[t]{2}{*}{$\begin{array}{l}\text { Prejuicio de } \\
\text { categoría }\end{array}$} & \multirow[t]{2}{*}{ Puntual } & \multicolumn{2}{|c|}{$\begin{array}{c}\text { Intervalo de } \\
\text { confianza } 95 \%\end{array}$} \\
\hline & & Mínimo & Máximo \\
\hline Muy en desacuerdo & 7,0 & 4,0 & 10,0 \\
\hline Algo en desacuerdo & 33,0 & 27,5 & 38,5 \\
\hline Algo de acuerdo & 45,0 & 39,2 & 50,8 \\
\hline Muy de acuerdo & 15,0 & 10,9 & 19,1 \\
\hline
\end{tabular}

Tabla 3. Prevalencia del prejuicio de categorías de la dimensión carácter-personalidad en personal de salud

\begin{tabular}{|c|c|c|c|}
\hline \multirow[t]{2}{*}{$\begin{array}{l}\text { Prejuicio de } \\
\text { categoría }\end{array}$} & \multirow[t]{2}{*}{$\%$} & \multicolumn{2}{|c|}{$\begin{array}{c}\text { Intervalo de confianza } \\
95 \%\end{array}$} \\
\hline & & Mínimo & Máximo \\
\hline Muy en desacuerdo & 10,5 & 6,9 & 14,1 \\
\hline Algo en desacuerdo & 25,5 & 20,4 & 30,6 \\
\hline Algo de acuerdo & 42,0 & 36,3 & 47,7 \\
\hline Muy de acuerdo & 22,0 & 17,2 & 26,8 \\
\hline
\end{tabular}

Tabla 4. Prevalencia del prejuicio de categorías de la evaluación global en personal de salud

\begin{tabular}{|lccc|}
\hline $\begin{array}{l}\text { Prejuicio de } \\
\text { categoría }\end{array}$ & $\%$ & \multicolumn{2}{c|}{$\begin{array}{c}\text { Intervalo de } \\
\text { confianza 95\% }\end{array}$} \\
\hline Muy en desacuerdo & 6,0 & 3,2 & 8,8 \\
Mínimo & Máximo \\
\hline Algo en desacuerdo & 29,0 & 23,7 & 34,3 \\
\hline Algo de acuerdo & 45,5 & 39,7 & 51.3 \\
\hline
\end{tabular}

Tabla 5. Prevalencia del estereotipo negativo por dimensión y global en personal de salud

\begin{tabular}{|lccc|}
\hline $\begin{array}{l}\text { Factores (dimensión) } \\
\text { y Global }\end{array}$ & $\%$ & \multicolumn{2}{c|}{$\begin{array}{c}\text { Intervalo de } \\
\text { confianza 95\% }\end{array}$} \\
Salud & 64,5 & 59,0 & 70,0 \\
Motivación-social & 60,0 & 54,3 & 65,7 \\
\hline Carácter personalidad & 64,0 & 58,4 & 69,6 \\
\hline Global & 65,0 & 59,5 & 70,5 \\
\hline
\end{tabular}

\section{Discusión}

El estereotipo hacia la vejez es parte de la cultura y por lo tanto, forma parte de la sociedad ${ }^{35,37}$. En la literatura los estudios sobre estereotipos de la vejez concluyen que la percepción social sobre las personas mayores es básicamente negativa ${ }^{24}$. En el área de la salud ha sido estudiado sólo en personal médico ${ }^{29,37}$, por lo que abordar el tema en el resto del personal, como se realiza en este estudio se traduce en aportación del conocimiento sobre el tema.

El cuestionario (CENVE) ${ }^{32}$ empleado en este trabajo para evaluar el estereotipo incorpora dos propuestas la de Montorio (Montorio e Izal 1991); que incluyen la dimensión salud, carácter-personalidad y motivación-social, se incorpora la propuesta de Palmore que incluye salud mental ${ }^{21}$, incorporándolo a la dimensión salud de Montorio. Convirtiéndolo en un instrumento integrador y representativo de los estereotipos negativos hacia la vejez descritos en la literatura.

Una de las características del estereotipo es la categorización ${ }^{15}$, la cual propicia la visión de grupo; la evaluación realizada en personal de salud corresponde al que labora en medio hospitalario, quien atiende adultos mayores con problemas crónicos agudizados, al que identifica como un ente aislado sin apoyo de redes sociales en consecuencia el estereotipo identificado solo aplica a este tipo de personal por lo que no es posible extrapolar a primer nivel, por lo que si se desea conocer el estereotipo en este personal es necesario la realización de otro estudio.

En la literatura se ha descrito que el estereotipo negativo en el personal médico se asocia con la calidad de la atención ${ }^{40}$, por lo que, la prevalencia global de $60 \%$ en este estudio puede tener implicaciones graves conside- 
Estereotipos negativos de la vejez en personal de salud en México - M. Franco S. et al

rando que la transición demográfica y epidemiológica implica mayor demanda de servicios de salud, con enfermedades crónico-degenerativas asociadas a períodos de hospitalización más prolongados.

El estereotipo negativo en la dimensión salud se comporta de manera semejante a otras dos dimensiones, debido a que es un componente cultural no racionalizado, que la formación profesional no logra modificarlo, lo cual es un grave problema ya que hace pensar que ésta se limita a crear técnicos y no profesionales en el sentido extenso del término.

Lo señalado evidencia la necesidad de un cambio de cultura, ya que la transición demográfica ha superado la visón del personal de salud, en el cual se encuentra ausente la perspectiva gerontológica, este proceso necesariamente pasará por una redefinición del significado social de la vejez y de las formas de integración social y cultural de los adultos mayores ${ }^{41}$.

En conclusión la prevalencia del estereotipo negativo de la vejez en el personal de salud estudiado es alta y motivo de preocupación por ser el profesional responsable de atención de este grupo y por las implicaciones sociodemográficas.

\section{Referencias}

1. Kofi A. Una sociedad para todas las edades. Segunda Asamblea Mundial sobre el Envejecimiento: Madrid. 2002. Disponible en: http://www.un.org/spanish/envejecimiento/dpi2230spa.htm [acceso 20 agosto 2009].

2. Consejo Nacional de la Población. 11 de julio día mundial de la población. 2004: 17. Disponible en: http:// www.conapo.gob.mx/prensa/carpeta2004.pdf

3. Levy BR. Mind matters: Cognitive and physical effects of aging self-stereotype. J Gerontol 2003; 58: 203-11.

4. Moragas R. Gerontología social. Envejecimiento y calidad de vida. Barcelona Herder; 1991.

5. Butler DM, Lewis MI. Agin and mental health: Positive psychosocial approaches. San Luis: C.V. Mosby; 1982.

6. Little A. Psychological aspects of working with elderly client. En A. J. Squires (ed.), Rehabilitation of the older patient. Sydney: Croom Helm; 1998.

7. Matras J. Dependency, Obligations and Entitlement: A New Sociology of Aging. Englewood Cliffs, N. J: Prentice-Hall; 1990.

8. Moñivas A. Representaciones de la vejez (modelos de disminución y de crecimiento). Anales de Psicología 1998; 14: 13-25.
9. Lippman W. Public Opinion. Free Press Paperbacks (originally published New York: Macmillan, 1922). New York; 1997.

10. Vinacke WE. Explorations in the dynamic processes of stereotyping. J Social Psychol 1956; 2: 105-32.

11. Oskamp S. Attitudes and opinions. Englewood Cliffs, 2a Ed., Prentice Hall; 1977.

12. Allport GW. The historical background of social psychology, in G. Lindsey y Aronson. Nueva York. The Handbook of Social Psychology 1987; 2: 34-7.

13. Tajfel H, Turner LC. La Teoría de la identidad social de la conducta intergrupal. En J.F. Morales y C. Huici, (Eds): Lecturas de Psicología Social. Madrid: UNED; 1989.

14. Miller AG. In the eye of the beholder. Conteporary issues in stereotyping. Nueva York; 1982. p. 25-7.

15. Echebarria A, González JL. Psicología social del prejuicio y del racismo. Madrid: Ramón Areces; 1996.

16. Brigham JC. Ethnic Stereotypes. Psychological Bolletin 1971; 76: 15-38.

17. Cano GJI. Los estereotipos sociales. El proceso de perpetuación a través de la memoria selectiva (tesis doctoral) Madrid: Universidad Complutense de Facultad de Sociología; 1993.

18. Wade S. Combating ageism: An imperative for contemporary health care. Rev Clin Gerontol 2001; 11: 285-94.

19. Tuckman J, Lorge I. Attitudes toward older workers. J Appl Psychol 1952; 36: 149-53.

20. Graham ID, Baker PM. Status, age and gender: perception of old and young people. Canad J Aging 1989; 8: 255-267.

21. Sánchez A. Imagen y Estereotipos de los ancianos en Venezuela. Revista Latinoamericana de Psicología 1982; 14: 363-83.

22. Hickey T, Hickey L, Kalish RA. Children's perceptions of the elderly. J Gen Psychol 1968; 112: 227-35.

23. Weinberger LE, Millham J. A multi-dimensional, multiple method analysis of attitudes toward the elderly. J Gerontol 1975; 30: 343-8.

24. Dulcey E, Ardila R. Actitudes hacia los ancianos. Revista Latinoamericana de Psicología 1976; 8: 57-67.

25. González FMA, Sánchez-Cifuentes MJ, Tonda E. González BP, Cid J. Estereotipos hacia los ancianos: Los ancianos vistos por otros grupos de edad. Revista Iberoamericana de Geriatría y Gerontología. Geriátrica 1990; 6: 204-9.

26. Molina del Peral JA. Estereotipos hacia los ancianos. Estudio comparativo de la, variable edad. Revista de Psicología General y Aplicada 2000; 53: 489-501.

27. Saarnio DA, Slotthebarnk CS. Putting attitudes toward the elderly in their place: Differences by domain of characteristics. Paper presented at the meeting of the 
Midwestern Psychological Association, Chicago; 1992.

28. Brown AS. The social processes of aging and old age: stereotypes in old age. New Jersey: Prentice Hall; 1996.

29. Amy JC, Cuddy AJC, Norton MI, Fiske ST. This old stereotype: the pervasiveness and persistence of the elderly stereotype. J Social Issues 2005; 61: 267-85.

30. Revenson. Compassionate stereotyping of elderly patients by physicians. Revising the Social Contact Hipothesis; (Psychology and aging); 1989.

31. Anantharaman RN. Perception of stereotypes towards old age by two generations. J Psycholo Researches 1984; 28: 170-3.

32. Blanca MJ, Sánchez CP, Trianes MV. Estereotipos negativos de la vejez. Rev Mult Gerontol 2005; 15: 212-20.

33. Palmore. First fact on aging quiz in a multiple-choice format. Educational Gerontology 1996; 6: 575-89.

34. Sáez N. Los estereotipos en los ancianos: Un estudio empírico y sus resultados. Revista de Psicología de la Educación 1994; 124: 75-89.

35. Hummert ML, Garstka TA, Shaner JL, Strahm S. Judg- ments about stereotypes of elderly. Research on Aging 1995; 2: 168-89.

36. Montorio I, Izal M, Sánchez M, Losada A. Dependencia y autonomía funcional en la vejez: La profecía que se autocumple. Revista Multidisciplinar de Gerontología 2002; 12: 61-71.

37. Montorio I. Actitudes hacia las personas mayores y discriminación basada en la edad. Revista Multidisciplinar de Gerontología 1998; 8: 21-30.

38. Montañés J, Latorre JM. Psicología de la vejez: estereotipos juveniles sobre el envejecimiento. Universidad de Castilla-La Mancha. De Cuenca Ediciones; 2004.

39. Avolio BJ, Barret GV. Effects of age stereotyping in a simulated interview. Psychol Aging 1987; 2: 56-63.

40. Levy BR, Banaji MR. Implicit ageism. En T. Nelson Ediciones. Stereotypes and prejudice against older persons Cambridge, MIT Press 2002; p. 49-75.

41. Edelstein BA, Martin RR, Koven LP. Psychological assessment in geriatric settings. En Graham JR y Naglieri JA. Editorial Handbook of Psychology 2003; 10: 389-414. 